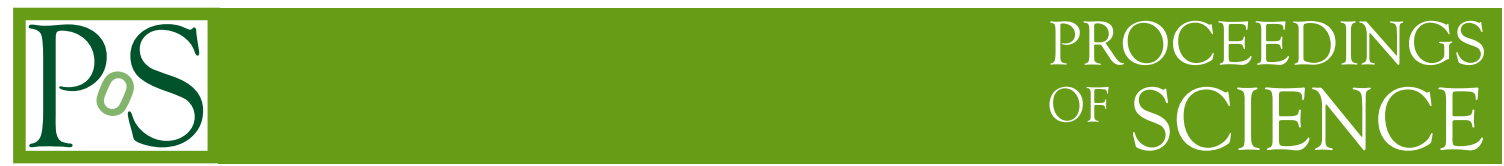

\title{
High-energy evolution of Wilson lines at the next-to-leading order
}

\author{
Giovanni Antonio Chirilli* \\ Department of Physics, The Ohio State University, Columbus, OH 43210, USA \\ E-mail: chirilli.1@osu.edu
}

\begin{abstract}
Proton-Nucleus, Nucleus-Nucleus and DIS scattering amplitudes at high-energy are described by matrix elements of Wilson line operators. The energy dependence of such amplitudes is described by the evolution equation of Wilson lines with respect to the rapidity parameter - the Balitsky-JIMWLK evolution equation. Most of the current phenomenology of high-energy and high-density QCD is based on the leading-order evolution equation with only running coupling corrections. In my talk I will present the derivation of the Balitsky-JIMWLK evolution equation at the next-to-leading order.
\end{abstract}

XXIII International Workshop on Deep-Inelastic Scattering,

27 April - May 12015

Dallas, Texas

${ }^{*}$ Speaker. 


\section{Introduction}

Relevant operators to describe scattering amplitudes at high energy are Wilson line operators: path-ordered exponential along the trajectory of the particle's velocity. In QCD the Wilson lines are defined as

$$
U\left(x_{\perp}\right)=1+i g \int_{-\infty}^{\infty} d u A_{\bullet}\left(u p_{1}+x_{\perp}\right)+(i g)^{2} \int_{-\infty}^{+\infty} d u \int_{-\infty}^{u} d v A_{\bullet}\left(u p_{1}+x_{\perp}\right) A_{\bullet}\left(v p_{1}+x_{\perp}\right)+\ldots
$$

Here, we used the notation $x_{\bullet}=\sqrt{\frac{s}{2}} x^{-}$and $x_{*}=\sqrt{\frac{s}{2}} x^{+}$with $x^{+}$and $x^{-}$light-cone coordinates defined as $x^{ \pm}=\frac{x^{0} \pm x^{3}}{\sqrt{2}} ; p_{1}^{\mu}$ and $p_{2}^{\mu}$ are ligh-cone vectors such that $p_{1}^{2}=p_{2}^{2}=0$ and $p_{1} \cdot p_{2}=\frac{s}{2}$ with $s$ the Mandelstam variable for the center-of-mass energy.

At High-energy scattering amplitudes can be expanded in terms of Wilson lines using the Operator Product Expansion (OPE) [1,2]. The energy dependence of the amplitudes is given by the evolution equations in rapidity of the Wilson line operators.

In high-energy Deep Inelastic Scattering (DIS) the virtual photon emitted by the lepton long before scattering off the hadronic target, splits into a quark and anti-quark pair. The propagation of the color dipole through the hadronic target is given by a scattering amplitude proportional to two Wilson lines. The amplitude can then be written as a convolution of the a coefficient function (the photon impact factor now known at NLO $[3,4]$ ) and a matrix element of a color dipole. The evolution equation of the color dipole represent the first of the Balitsky hierarchy of evolution equations which, in the large $N_{c}$ approximation, reduces to the Balitsky-Kovchegov equation $[1,5]$ (for a review see Ref. [6]).

For proton-Nucleus or Nucleus-Nucleus collisions, the scattering amplitudes are composed of several Wilson lines and the corresponding evolution equation is the Balitsky-JIMWLK evolution equation $[1,7]$.

Before we present the result for the NLO evolution equation of Wilson lines in Section 3, let us begin with a brief pedagogical introduction to the background field technique in Section 2, and derive as an example the evolution of one Wilson line operator. In section 3 we will present the NLO correction to the evolution equation of one Wilson line and the evolution equation of Wilson lines with triple interactions. The complete result of the Balitsky-JIMWLK evolution equation can be found in Ref. [8].

\section{Leading Order Evolution Equation}

In this section we provide an introduction to the background field method used to derive the evolution equation of Wilson line operators.

At high-energy (high-parton density) the energy dependence of the scattering amplitude is encoded in the evolution of matrix elements made of Wilson lines. Let us indicate with $\mathscr{O}^{\eta_{1}}$ an operator made of several Wilson lines with rapidity dependence $\eta_{1}$ and if we indicate with $|B\rangle$ the target state, then the scattering amplitude is proportional to

$$
\left\langle B\left|\mathscr{O}^{\eta_{1}}\right| B\right\rangle
$$

The Wilson lines may depend on the rapidity parameter in at least two different ways. One way is the dependence by slope: if the particle propagate at infinite energy then its trajectory is on 
the light cone. If we assume that the energy is very large but not infinite, then, the trajectory of the particle is slightly off the light cone i.e it is along $n^{\mu}=p_{1}^{\mu}+e^{-2 \eta} p_{2}^{\mu}$ direction. The energy of the particle is given by its rapidity $\eta$ and when the energy is infinite the propagation of the particle is parallel to the light-cone vector $p_{1}^{\mu}$.

The rapidity dependence by slope of the Wilson line is

$$
U^{\eta}\left(x_{\perp}\right)=\operatorname{Pexp}\left\{i g \int_{-\infty}^{\infty} d u n_{\mu} A^{\mu}\left(u n+x_{\perp}\right)\right\}
$$

Alternatively, one can include the energy dependence into the Wilson line operator by rigid cut-off i.e. by cutting off the longitudinal component of the momentum of the gluon in the following way

$$
U_{x}^{\eta}=\operatorname{Pexp}\left[i g \int_{-\infty}^{\infty} d u p_{1}^{\mu} A_{\mu}^{\eta}\left(u p_{1}+x_{\perp}\right)\right]
$$

with

$$
A_{\mu}^{\eta}(x)=\int \frac{d^{4} k}{(2 \pi)^{4}} \theta\left(e^{\eta}-\left|\alpha_{k}\right|\right) e^{-i k \cdot x} A_{\mu}(k)
$$

and with $k^{\mu}=\alpha_{k} p_{1}^{\mu}+\beta_{k} p_{2}^{\mu}+k_{\perp}^{\mu}$. As it has been shown in Ref. [11,8], at NLO it is more convenient to use the rapidity dependence by rigid cut-off.

At high energy the main degree of freedom are gluons, therefore we may assume, in the first approximation, that the target is made of gluon field. Let us then consider the operator $\mathscr{O}^{\eta_{1}}$ in the background of an external gluonic field and calculate its evolution with respect to the rapidity parameter by changing the parameter $\eta_{1}$ by an infinitesimal step $\Delta \eta$. To this end, we introduce a rapidity divide $\eta_{2}$ which separates the classical fields having rapidity up to $\eta_{2}$ from the quantum fields having rapidity $\eta_{1}>\eta>\eta_{2}$ that will be integrated out to form Feynman diagrams. Formally, the separation of the correlation function in classical and quantum fields may be written as follows

$$
\left\langle\mathscr{O}^{\eta_{1}}\right\rangle_{A} \rightarrow\left\langle\mathscr{O}^{\prime \eta_{2}} \otimes \mathscr{O}^{\prime \eta_{1}}\right\rangle_{A}
$$

The subscript $A$ indicates that the matrix elements are evaluated in the background of the gluonic external field. In principle, after the separation of the fields in classical and quantum components, the operator may be different from the one we started with. We have indicated this with a prime on the operator $\mathscr{O}$ in Eq. (2.5). Since particles with different rapidities perceive each other as Wilson lines, the operators obtained after splitting the fields in classical and quantum are still Wilson lines.

The result of the integration of the matrix element on the right-hand-side (RHS) of Eq. (2.5) over the quantum fields is the kernel of the one loop evolution equation times the matrix element of the operator made of the classical fields i.e. with rapidity parallel to $\eta_{2}$, and times the infinitesimal step in rapidity $\Delta \eta=\eta_{2}-\eta_{1}$. The one-loop evolution equation of the $\mathscr{O}$ operator with respect to rapidity is

$$
\left\langle\mathscr{O}^{\eta_{1}}\right\rangle_{A}=\alpha_{s}\left(\eta_{1}-\eta_{2}\right) K_{\mathrm{evol}} \otimes\left\langle\mathscr{O}^{\prime \eta_{2}}\right\rangle_{A}
$$

The resulting evolution equation, obtained following the semi-classical approach just described, can be linear or non-linear: 
- Linear case $\mathscr{O}^{\eta_{1}}=\alpha_{s} \Delta \eta K_{\text {evol }} \otimes \mathscr{O}^{\eta_{2}}$

- Non-linear case $\mathscr{O}^{\eta_{1}}=\alpha_{s} \Delta \eta K_{\text {evol }} \otimes\left\{\mathscr{O}^{\eta_{2}} \mathscr{O}^{\eta_{2}}\right\}$

The rapidity evolution of one Wilson line is given by the Feynman diagrams in Fig. (2). The red strip in the figure represents the background field in the spectator frame: the external field is highly boosted, and it gets contracted in the direction of the boost and time-dilated. Thus, the propagation of the particle is in the background of a shock-wave external field. The evolution

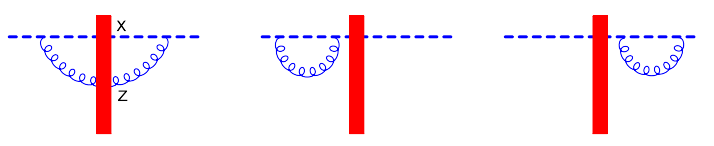

Figure 1: Feynman diagrams for one loop evolution of one Wilson lines.

equation for one Wilson line is

$$
\left\langle\left\{U_{x}^{\eta_{1}}\right\}_{i j}\right\rangle_{A}=\frac{\alpha_{s}}{2 \pi^{2}} \Delta \eta \int \frac{d^{2} z}{(x-z)_{\perp}^{2}}\left[\left\langle\operatorname{tr}\left\{U_{x}^{\eta_{2}} U_{z}^{\eta_{2} \dagger}\right\}\left\{U_{z}^{\eta_{2}}\right\}_{i j}\right\rangle_{A}-\left\langle\frac{1}{N_{c}}\left\{U_{x}^{\eta_{2}}\right\}_{i j}\right\rangle_{A}\right]
$$

where we have used the short-hand notation $U_{x} \equiv U\left(x_{\perp}\right)$, and the color index $i, j=1,2,3$. The kernel of the evolution in this case is $K(x, z)=\frac{1}{(x-z)^{2}}$, and the equation is clearly non-linear. Before the one-loop evolution, we have one Wilson line with rapidity $\eta_{1}$ corresponding to the propagation of one quark in the background of a shock-wave. At one loop order, instead, we have a quark and a gluon propagating in the shock-wave.

The scattering amplitude of DIS is proportional to

$$
\mathscr{U}\left(x_{\perp}, y_{\perp}\right)=1-\frac{1}{N_{c}} \operatorname{tr}\left\{U\left(x_{\perp}\right) U^{\dagger}\left(y_{\perp}\right)\right\}
$$

To get the evolution equation of the color-dipole we need the evolution equation of the operator $U_{x}$ and $U_{y}^{\dagger}$, and also the evolution equation of pairwise interactions. Thus, the operator $\frac{d}{d \eta}$ of the evolution equation does not follow the Leibniz rule for derivative of product of function. Indeed, this is represented by the Feynman diagrams in Fig. (2),

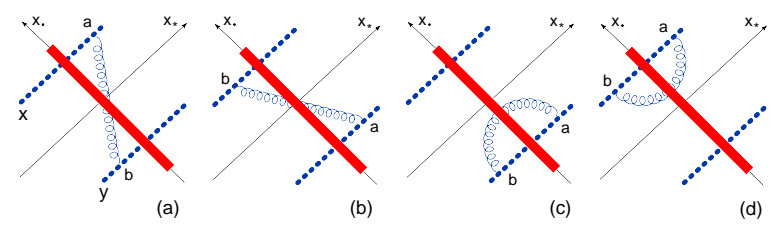

Figure 2: Feynamn diagram for the LO evolution equation of a color-dipole. We omitted the virtual correction diagrams.

and the corresponding evolution equation is

$$
\frac{d}{d \eta} \hat{\mathscr{U}}(x, y)=\frac{\alpha_{s} N_{c}}{2 \pi^{2}} \int \frac{d^{2} z(x-y)^{2}}{(x-z)^{2}(y-z)^{2}}\{\hat{\mathscr{U}}(x, z)+\hat{\mathscr{U}}(z, y)-\hat{\mathscr{U}}(x, y)-\hat{\mathscr{U}}(x, z) \hat{\mathscr{U}}(z, y)\}
$$


Equation (2.9) is the LO Balitsky evolution equation [1] for color dipole. When the non-linear term operator $\langle\hat{\mathscr{U}}(x, z) \hat{\mathscr{U}}(z, y)\rangle$ factorizes at large $N_{c}$ as $\langle\hat{\mathscr{U}}(x, z)\rangle\langle\hat{\mathscr{U}}(z, y)\rangle$, Eq. (2.9) becomes the Balitsky-Kovchegov equation [1,5]. The linear terms in Eq. (2.9) correspond to the BFKL evolution equation obtained in perturbative QCD in the leading-log resummation $\alpha \ll 1$ and $\alpha_{s} \eta \sim 1$; while the non-linear term appears because of the semi-classical approach where the new resummation parameter is $\alpha_{s}^{2} A^{1 / 3}$ with $A$ being the atomic number in the case of DIS off a nuclear target. The BFKL equation is known to violate unitarity, but the non linear term in Eq. (2.9) preserves unitarity.

In order to get the LO evolution equation for trace of any number of Wilson lines or product of any number of Wilson lines, which would correspond to the Balitsky-JIMWLK evolution equation $[1,7]$, one needs to obtain the evolution equation for the following operators as well: $\left\{U_{x}^{\dagger \eta_{1}}\right\}_{i j},\left\{U_{x}^{\eta_{1}} U_{y}^{\eta_{1}}\right\}_{i j},\left\{U_{x}^{\eta_{1}} U_{y}^{\dagger \eta_{1}}\right\}_{i j},\left\{U_{x}^{\dagger \eta_{1}} U_{y}^{\dagger \eta_{1}}\right\}_{i j}$. Thus, one obtains a set of five evolution equations that can be used to calculate the evolution equation of operators with any trace of Wilson lines. As an example, let us consider the evolution equation of a four Wilson lines operator $\operatorname{tr}\left\{U_{x} U_{y}^{\dagger} U_{w} U_{z}^{\dagger}\right\}$. To this end, one has to sum the evolution of each single Wilson line using the evolution equation like Eq. (2), and similar evolution equations for each paring.

Our ext task is to obtain the evolution equations of matrix elements of operators with any trace of Wilson lines at NLO. We will obtain evolution equations similar to the one given in (2.7) but at the next-to-leading order (NLO).

\section{Next-to-leading order evolution equation}

In order to obtain the evolution equation of operators with any trace of Wilson lines (or also product of Wilson lines) at the next to leading order, one has to calculate a similar set of evolution equations at NLO. At NLO, however, we may have not only the evolution of single Wilson lines and of two connected Wilson lines but also evolution of triple Wilson lines interaction (see Fig. 3 e) and f)). The diagrams contributing to the NLO B-JIMWLK with three Wilson lines were calculated
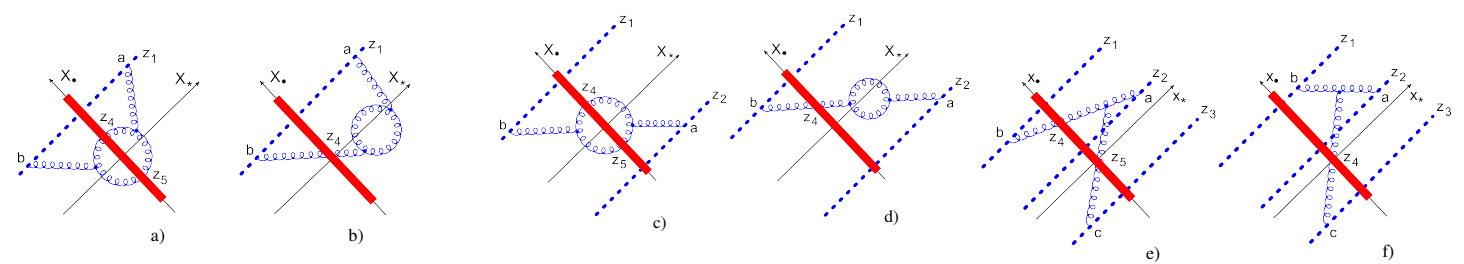

Figure 3: Sample of Feynman diagrams contributing to the NLO B-JIMWLK evolution equation.

in Ref. [9]. In Ref. [8] we have calculated the full Balitsky hierarchy at NLO, confirming also the result obtained in Ref. [9]. The NLO JIMWLK Hamiltonian [10], on the other hand, was obtained using the NLO BK equation calculated in Ref. $[11,12]$ and the evolution with three connected Wilson lines of Ref. [9].

For simplicity, we present here only the one-particle interaction ("gluon reggeization" term) at 
NLO

$$
\begin{aligned}
& \frac{d}{d \eta}\left(U_{1}\right)_{i j}=\frac{\alpha_{s}^{2}}{8 \pi^{4}} \int \frac{d^{2} z_{4} d^{2} z_{5}}{z_{45}^{2}}\left\{U _ { 4 } ^ { d d ^ { \prime } } ( U _ { 5 } ^ { e e ^ { \prime } } - U _ { 4 } ^ { e e ^ { \prime } } ) \left(\left[2 I_{1}-\frac{4}{z_{45}^{2}}\right] f^{a d e} f^{b d^{\prime} e^{\prime}}\left(t^{a} U_{1} t^{b}\right)_{i j}+\frac{\left(z_{14}, z_{15}\right)}{z_{14}^{2} z_{15}^{2}} \ln \frac{z_{14}^{2}}{z_{15}^{2}}\right.\right. \\
& \left.\left.\times\left[i f^{a d^{\prime} e^{\prime}}\left(\left\{t^{d}, t^{e}\right\} U_{1} t^{a}\right)_{i j}-i f^{a d e}\left(t^{a} U_{1}\left\{t^{d^{\prime}}, t^{e^{\prime}}\right\}\right)_{i j}\right]\right)\right\} \\
& +\frac{\alpha_{s}^{2} N_{c}}{4 \pi^{3}} \int d^{2} z_{4} z_{14}^{2}\left(U_{4}^{a b}-U_{1}^{a b}\right)\left(t^{a} U_{1} t^{b}\right)_{i j}\left\{\left[\frac{11}{3} \ln z_{14}^{2} \mu^{2}+\frac{67}{9}-\frac{\pi^{2}}{3}\right]\right\}
\end{aligned}
$$

where

$$
I_{1} \equiv I\left(z_{1}, z_{4}, z_{5}\right)=\frac{\ln z_{14}^{2} / z_{15}^{2}}{z_{14}^{2}-z_{15}^{2}}\left[\frac{z_{14}^{2}+z_{15}^{2}}{z_{45}^{2}}-\frac{\left(z_{14}, z_{15}\right)}{z_{14}^{2}}-\frac{\left(z_{14}, z_{15}\right)}{z_{15}^{2}}-2\right]
$$

and the evolution for triple interactions (a sample of diagrams is given in Fig. 3 e and f).

$$
\begin{aligned}
& \frac{d}{d \eta}\left(U_{1}\right)_{i j}\left(U_{2}\right)_{k l}\left(U_{3}\right)_{m n}=i \frac{\alpha_{s}^{2}}{2 \pi^{4}} \int d^{2} z_{4} d^{2} z_{5}\left\{\mathscr{J}_{12345} \ln \frac{z_{34}^{2}}{z_{35}^{2}}\right. \\
& \times f^{c d e}\left[\left(t^{a} U_{1}\right)_{i j}\left(t^{b} U_{2}\right)_{k l}\left(U_{3} t^{c}\right)_{m n}\left(U_{4}-U_{1}\right)^{a d}\left(U_{5}-U_{2}\right)^{b e}\right. \\
& \left.-\left(U_{1} t^{a}\right)_{i j}\left(U_{2} t^{b}\right)_{k l}\left(t^{c} U_{3}\right)_{m n}\left(U_{4}-U_{1}\right)^{d a}\left(U_{5}-U_{2}\right)^{e b}\right] \\
& +\mathscr{J}_{32145} \ln \frac{z_{14}^{2}}{z_{15}^{2}} f^{a d e}\left[\left(U_{1}^{\dagger} t^{a}\right)_{i j}\left(t^{b} U_{2}\right)_{k l}\left(t^{c} U_{3}\right)_{m n}\left(U_{4}-U_{3}\right)^{c d}\left(U_{5}-U_{2}\right)^{b e}\right. \\
& \left.-\left(t^{a} U_{1}\right)_{i j} \otimes\left(U_{2} t^{b}\right)_{k l}\left(U_{3} t^{c}\right)_{m n}\left(U_{4}^{d c}-U_{3}^{d c}\right)\left(U_{5}^{e b}-U_{2}^{e b}\right)\right] \\
& +\mathscr{J}_{13245} \ln \frac{z_{24}^{2}}{z_{25}^{2}} f^{b d e}\left[\left(t^{a} U_{1}\right)_{i j}\left(U_{2}^{\dagger} t^{b}\right)_{k l}\left(t^{c} U_{3}\right)_{m n}\left(U_{4}-U_{1}\right)^{a d}\left(U_{5}-U_{2}\right)^{c e}\right. \\
& \left.-\left(U_{1} t^{a}\right)_{i j}\left(t^{b} U_{2}\right)_{k l}\left(U_{3} t^{c}\right)_{m n}\left(U_{4}-U_{1}\right)^{d a}\left(U_{5}-U_{3}\right)^{e c}\right]
\end{aligned}
$$

where

$$
\begin{aligned}
& \mathscr{J}_{12345} \equiv \mathscr{J}\left(z_{1}, z_{2}, z_{3}, z_{4}, z_{5}\right)=-\frac{2\left(z_{14}, z_{34}\right)\left(z_{25}, z_{35}\right)}{z_{14}^{2} z_{25}^{2} z_{34}^{2} z_{35}^{2}} \\
& -\frac{2\left(z_{14}, z_{45}\right)\left(z_{25}, z_{35}\right)}{z_{14}^{2} z_{25}^{2} z_{35}^{2} z_{45}^{2}}+\frac{2\left(z_{25}, z_{45}\right)\left(z_{14}, z_{34}\right)}{z_{14}^{2} z_{25}^{2} z_{34}^{2} z_{45}^{2}}+\frac{\left(z_{14}, z_{25}\right)}{z_{14}^{2} z_{25}^{2} z_{45}^{2}}
\end{aligned}
$$

Here we have used the short-hand notation $U_{1} \equiv U\left(z_{1}\right)$ etc. In Eq. (3.1) we have omitted the quark-loop contributions. The evolution for pairwise interactions at NLO can be found in Ref. [8].

\section{Conclusions}

The factorization in rapidity of scattering amplitudes using the OPE at high-energy in Wilson lines is a successful tool for the study of processes at high energy. Its validity has been proven at NLO accuracy $[3,4]$. The BK equation is a large $N_{c}$ limit of the first of the Balitsky hierarchy equations. In order to generalize NLO BK equation to the NLO evolution of any number of Wilson lines, we considered the triple interaction besides the self-energy interaction and the pairwise interaction. For simplicity, here we have presented only the NLO evolution equation for self-energy 
interactions given in Eq. (3.1) and the evolution equation of Wilson lines with triple interactions given in Eq. (3.2). The evolution equation for pairwise interactions at NLO can be found in Ref. [8].

This material is based upon work supported by the U.S. Department of Energy, Office of Science, Office of Nuclear Physics under Award Number DE-SC0004286.

\section{References}

[1] I. Balitsky, Nucl. Phys. B 463, 99 (1996) [hep-ph/9509348].

[2] I. Balitsky and G. A. Chirilli, Phys. Lett. B 687, 204 (2010) [arXiv:0911.5192 [hep-ph]].

[3] I. Balitsky and G. A. Chirilli, Phys. Rev. D 87, 014013 (2013) [arXiv:1207.3844 [hep-ph]].

[4] I. Balitsky and G. A. Chirilli, Phys. Rev. D 83, 031502 (2011) [arXiv:1009.4729 [hep-ph]].

[5] Y. V. Kovchegov, Phys. Rev. D 60, 034008 (1999) [hep-ph/9901281].

[6] I. Balitsky, In *Shifman, M. (ed.): At the frontier of particle physics, vol. 2* 1237-1342 [hep-ph/0101042].

[7] J. Jalilian Marian, A. Kovner, A.Leonidov and H. Weigert, Nucl. Phys. B504, 415 (1997), Phys. Rev. D59, 014014 (1999); J. Jalilian Marian, A. Kovner and H. Weigert, Phys. Rev. D59, 014015 (1999); A. Kovner and J.G. Milhano, Phys. Rev. D61, 014012 (2000); A. Kovner, J.G. Milhano and H. Weigert, Phys. Rev. D62, 114005 (2000); H. Weigert, Nucl. Phys. A703, 823 (2002); E.Iancu, A. Leonidov and L. McLerran, Nucl. Phys. A692, 583 (2001), Phys. Lett. B510, 133 (2001); E. Ferreiro, E. Iancu, A. Leonidov, L. McLerran, Nucl. Phys. A703, 489 (2002).

[8] I. Balitsky and G. A. Chirilli, Phys. Rev. D 88, 111501 (2013) [arXiv:1309.7644 [hep-ph]].

[9] A. V. Grabovsky, JHEP 1309, 141 (2013) [arXiv:1307.5414 [hep-ph]].

[10] A. Kovner, M. Lublinsky and Y. Mulian, Phys. Rev. D 89, 061704 (2014) [arXiv:1310.0378 [hep-ph]].

[11] I. Balitsky and G. A. Chirilli, Phys. Rev. D 77, 014019 (2008) [arXiv:0710.4330 [hep-ph]].

[12] I. Balitsky and G. A. Chirilli, Nucl. Phys. B 822, 45 (2009) [arXiv:0903.5326 [hep-ph]]. 